\title{
Development of a Portable Sensorised Handle for the Objective Assessment of the Effectiveness and Concordance of Intervention Plans in Dementia
}

\author{
J. Ma, M. Zecca, Member, IEEE
}

\begin{abstract}
Dementia is a global issue which is rising rapidly, with 46.8 million people worldwide living with dementia in 2015. This number is expected to increase to 74.7 million by 2030 . Some researches show that some kinds of exercises, such as resistance band exercises, would help to improve the memory and other abilities in patients with dementia. However, the true effectiveness of these activities is not really known due to the lack of objective assessment of efforts done during the long intervention time. Moreover, there is no way of measuring the actual activities performed by the patient without direct observation. As a consequence, it is impossible to measure the concordance between the patient and the therapist.

For this purpose, we developed a sensorised handle to objectively measure and assess activities with the resistance bands. The small circuit board design can easily fit the same form factor of a standard handle. The low power design, embedded li-ion battery and on-board battery charger allow easy and long-time usage.
\end{abstract}

This paper presents the initial experimental evaluation of the sensorised resistance band handle.

\section{INTRODUCTION}

Dementia is a rising global issue, with annual global costs in excess of US\$ 800 billion [1]. 46.8 million people worldwide living with dementia in 2015, and this number is expected to increase to 74.7 million by 2030 . Dementia affects people differently, especially in early stage; how others respond to the person and how much support they receive from the surrounding environment and people will greatly affect their living quality [2]. At present the diagnosis is usually based on observation of GPs (General practitioner) and specialists [3], with lots of variability and errors.

Many authors have proposed that participating in physical and cognitive stimulating activities may improve memory and other cognitive abilities [4], in particular with resistance bands exercises [5].

To ensure the exercise plan is suited to for the patient, the exercises need to be quantified objectively, by recording and calculating the time and effort done by the subject. According to the previous research, the adherence (i.e. the majority of subjects follow the designed exercise plan) is always a

*Research partially supported by the HEFCE Catalyst grant and by the EESE startup grant.

J. Ma is with the Wolfson School of Mechanical, Electrical and Manufacturing Engineering, Loughborough University, UK.

M. Zecca is with the Wolfson School of Mechanical, Electrical and Manufacturing Engineering, Loughborough University, UK, and with the National Centre for Sport and Exercise Medicine - East Midlands, Loughborough, Leicestershire LE11 3TU UK; Phone: +44(0) 1509 227122; contact email address: m.zecca@lboro.ac.uk difficult issue for long term off-lab experiments, and getting worst in daily exercises monitoring [4]. When people is doing excises in home, the concordance (which means not only the agreement but also the harmony in the relationship of patient and therapist) between agreed plans and actual effort cannot be assessed. As a consequence, it is impossible to measure the concordance between the patient and the therapist.

However, it is very difficult to measure the actual activities performed by the patient without direct observation, in particular outside of a lab. The measuring instruments are always being set in lab-based environment. Obviously, the lab-based environment is not suitable for performing day-to-day exercise, especially when doing exercise is part of a long term intervention plan [4]. The resistance band is a portable exercise equipment, also easy to store and use in tight space or gym and are widely used in daily exercise.

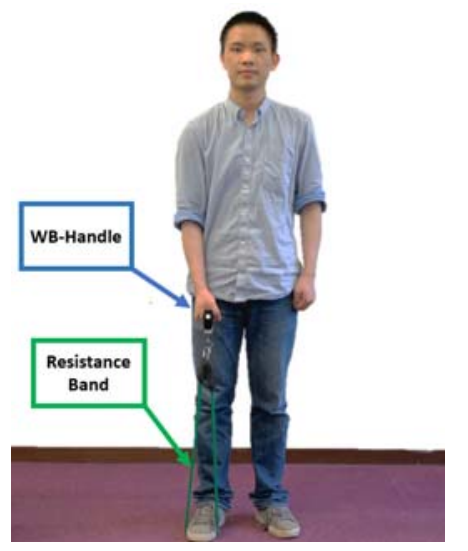

Fig. 1: Example of exercise with resistance bands

An example of resistance band exercise is shown in Fig. 1. The band is fixed by foot and the resistance band is pull by arm from waist to shoulder. There are various resistance level and exercises that can could be chosen, which makes the resistance band a good equipment for home exercises. However, there are many limitations of the finding. In particular:

- $\quad$ self-reporting of the exercises and activity levels done by subject is highly unreliable [6]

- there is no objective measurement and quantification of the exercises.

If the exercises done on the resistance band could be measured, which will be a potential way to help for quantify 
the exercise. One method is integrating electronics into the handle of the resistance band, to sense the motion of the handle based on Inertial Measurement Unit (IMU), and other information, such as the force applied on the resistance band. There are a few commercial IMU based sensing system on the market, like products from Xsens and the lab based system, WB-System developed by Waseda University. Both require professional skills for setting up and using [7] [8], which makes them impossible for daily exercise monitoring. The lack of force measurement abilities also limited the quantification of exercise, due to the unknown of the forces applied on the resistant band [8].

This paper presents the development of a novel sensor for elastic resistance bands, named WB-Handle based on IMU, barometer, and load cell, and its preliminary evaluation for the objective assessment of exercises.

\section{MATERIALS AND METHODS}

\section{A. WBR-SH1 sensoried handle design}

The new WBR-SH1 is developed specifically to objectively assess resistance band exercises.

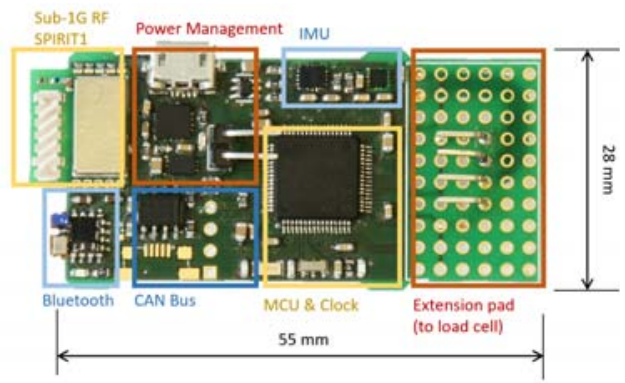

Fig. 2 Board layout (Front side)

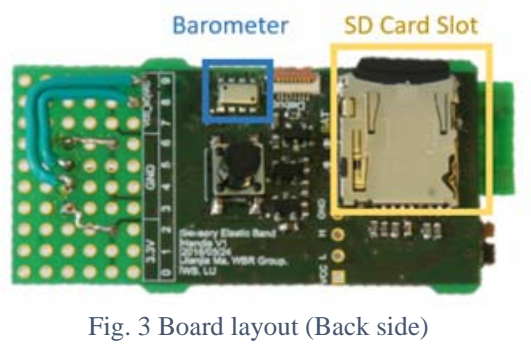

A single board design is applied to the circuit board to achieve small and compact design (Fig. 2 and Fig. 3). The board currently integrates a CORTEX-M microcontroller STM32F405RGT6 (in low power configuration, the more advanced low power microcontroller STM32L476RGT6 can be used,), a USB power management circuit, Bluetooth 4.2 module DA14580, SPIRIT1 Sub-1GHz wireless module and the following sensors: I-NEMO digital 3-Axis gyroscope and 3-Axis accelerometer module LSM6DS3; 3-Axis magnetometer LIS3MDL; and Barometer MS5611. A 6x10 pin extension pad (with common $2.54 \mathrm{~mm}$ pitch) is also on board with 10 programmable GPIOs and power ports. The load cell driver is connected to the board through this extension area. The major characteristics of the on-board sensors in the resistance band handle are listed in TABLE I. The settings used in current design is highlighted.
TABLE I MAIN CHARACTERISTICS OF THE WBR-SH1

\begin{tabular}{|c|c|c|c|c|}
\hline & \multicolumn{4}{|c|}{ Sensors } \\
\hline & \multicolumn{2}{|c|}{ LSM6DS3 } & \multirow{2}{*}{$\begin{array}{c}\text { LIS3MDL } \\
\begin{array}{c}\text { Magneto-m } \\
\text { eter }\end{array}\end{array}$} & \multirow{2}{*}{$\begin{array}{c}\text { MS5611 } \\
\text { Barometer }\end{array}$} \\
\hline Category & Gyroscope & $\begin{array}{c}\text { Accelero- } \\
\text { meter }\end{array}$ & & \\
\hline Axis & 3 -axis & 3 -axis & 3 -axis & N/A \\
\hline Size & \multicolumn{2}{|c|}{$2.5 \times 3 \times 0.83[\mathrm{~mm}]$} & $2 \times 2 x 1[\mathrm{~mm}]$ & $5 \times 3 \times 1[\mathrm{~mm}]$ \\
\hline Range & $\begin{array}{c} \pm 125 / \\
\pm 250 / \\
\pm 500 / \\
\pm \mathbf{1 0 0 0} / \\
\pm 2000 \text { [dps] }\end{array}$ & $\begin{array}{l} \pm 2 / \\
\pm 4 / \\
\pm 8 / \\
\pm 16 \\
{[G]}\end{array}$ & $\begin{array}{c} \pm 4 / \\
\pm 8 / \\
\pm 12 / \\
\pm 16[\text { Gauss }]\end{array}$ & $\begin{array}{c}450 \text { to } 1100 \\
\text { [mBar] }\end{array}$ \\
\hline Resolution & 16 [bit] & 16 [bit] & 16 [bit] & 24 [bit] \\
\hline Bandwidth & $830[\mathrm{~Hz}]$ & $3.3 \mathrm{k}[\mathrm{Hz}]$ & $500[\mathrm{~Hz}]$ & $250[\mathrm{~Hz}]$ \\
\hline Current & $1.25[\mathrm{~mA}]$ & $0.24[\mathrm{~mA}]$ & $0.27[\mathrm{~mA}]$ & $1.4[\mathrm{~mA}]$ \\
\hline
\end{tabular}

The configuration block diagram of the WB-Handle is shown in Fig. 4.

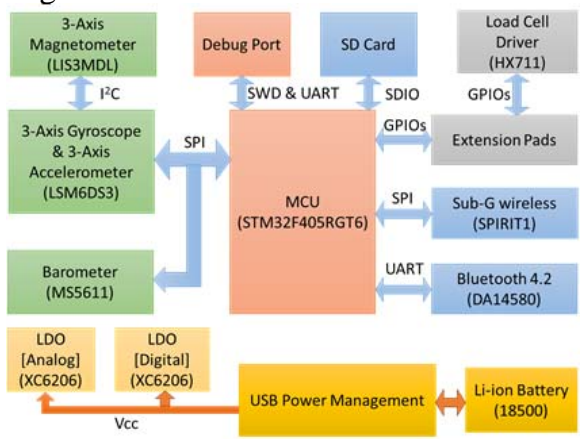

Fig. 4 Configuration block diagram of WBR-SH1

The handle directly measures the 3D angular velocity and 3D acceleration by 3-Axis gyroscope and 3-Axis accelerometer. In addition, the load cell provides the force applied to the resistance band.

\section{B. Experiment}

\section{1) Experimental setup}

The experimental setup is shown in Fig. 5. The traditional handle (non-sensory) is replaced by the WBR-SH1 . The WBR-SH1 collects motion data (acceleration, angular rotations), magnetic data, air pressures and the force applied on the resistance band. When subjects are doing exercise, the data is being recorded in to the micro SD card and sent to PC by wireless communication.

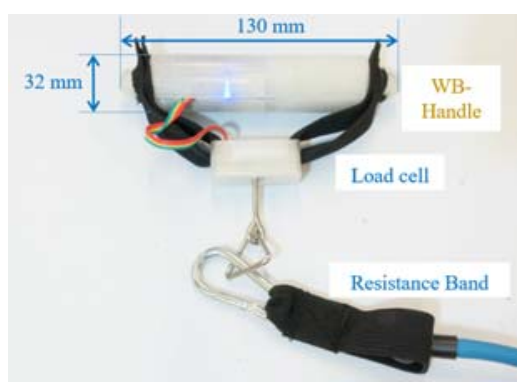

Fig. 5 Experiment set-up 


\section{2) Experimental protocol}

One preliminary experiment was performed by one healthy volunteer, male, age 24, right handed. Fig. 1 shows the experimental set-up and placement of the sensor. The subject was asked to do the arm curl muscle test in 30 seconds with both hands and 2 types of resistance band (2 different levels of resistances). The subject was asked to complete every cycle by pulling the resistance band from waist to shoulder then to release. In between of each test, there were 10 minutes' interval for rest and recovery to avoid the effects of fatigue.

\section{3) Data pre-processing}

During the experiment, all data are acquired at sample frequency $f=1000 \mathrm{~Hz}$ at and then stored in different CSV files on the PC. The accelerations, angular velocity, magnetic field, air pressure and load cell data is low pass filtered by a moving windows average filter of $10 \mathrm{~Hz}$ to remove measurement noise and artefacts. In this paper, the data of gyroscope and load cell are used for data processing.

\section{4) Calibration}

For the gyroscope calibration, the calibration process is similar with conventional 3-Axis gyroscope[10]. Ignoring the misalignment error, the gyroscope can be descripted as the model below:

$$
\left[\begin{array}{l}
d_{m x} \\
d_{m y} \\
d_{m z}
\end{array}\right]=\left[\begin{array}{ccc}
G_{x} & 0 & 0 \\
0 & G_{y} & 0 \\
0 & 0 & G_{z}
\end{array}\right]\left(\left[\begin{array}{l}
d_{r x} \\
d_{r y} \\
d_{r z}
\end{array}\right]-\left[\begin{array}{l}
b_{x} \\
b_{y} \\
b_{z}
\end{array}\right]\right)
$$

In (1), $d_{m x}, d_{m y}, d_{m z}$ represent the idea measurement result of the sensor; $d_{r x}, d_{r y}, d_{r z}$ represent the actual output by the sensor. $G_{x}, G_{y}, G_{z}$ are the gains of each axis; $b_{x}, b_{y}, b_{z}$ are the bias of each axis. The calibration of gyroscope is divided to 2 steps. The first step will measure the biases of each axis by setting the sensor still for a short time. The second step calculate the gains. A turntable is introduced which is able to provides stable rotation as reference.

The load cell is based on full-bridge resistance network and it is measure by a specific weight driver (HX711). It is possible to model load cell sensor by (2).

$$
d_{m}=G\left(d_{r}-b\right)
$$

$d_{m}$ represents the ideal result by load cell sensor; $d_{r}$ is the real output of the sensor. $G$ is the gain and $b$ is the bias. The calibration divided to 2 step: the first step will put the sensor in idle to measure the bias; the second step applies a fixed force on the load cell then the gain can be calculated.

\section{EXPERIMENTAL RESULTS}

The preliminary data processing focuses on the load cell data, which records the force loaded on resistance band. The data is segmented by using gyroscope for each repetition.

The gyroscope output represents the rotation on axis of the WBR-SH1. Each repetition is identified by the zero-crossing of the gyroscope data from negative to positive (indicated by the red vertical lines in Fig. 6), which corresponds to the peak force of each repetition. The force data are segmented to each repetition by using the middle point between two repetitions' $10 \%$ of peak force points.
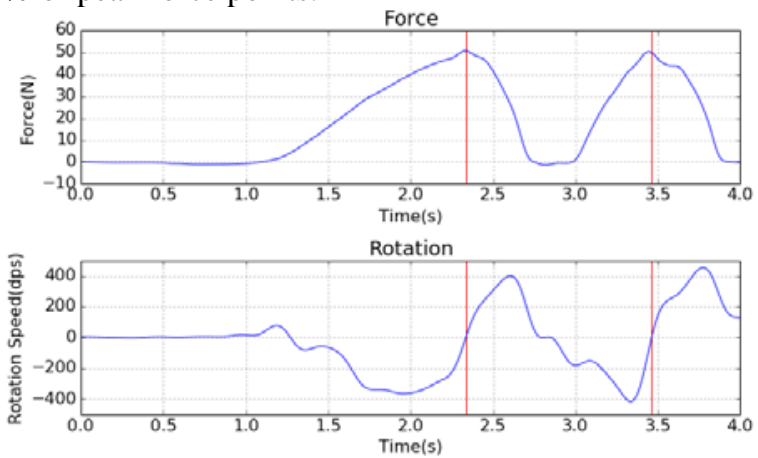

Fig. 6 Right hand yellow band force and gyroscope data

In each repetition, 3 types of interesting timing are plotted: the rising time $\left(t_{\text {rise }}\right)$ from $10 \%$ to $90 \%$ of the peak force of that cycle; the falling time $\left(t_{\text {fall }}\right)$ from $90 \%$ to $10 \%$ of peak force; and the total time of each repetition ( $T_{\text {duration }}$ ) measured by from $10 \%$ of peak force in pulling to $10 \%$ of peak force in falling. These parameters are graphically shown in Fig. 7.

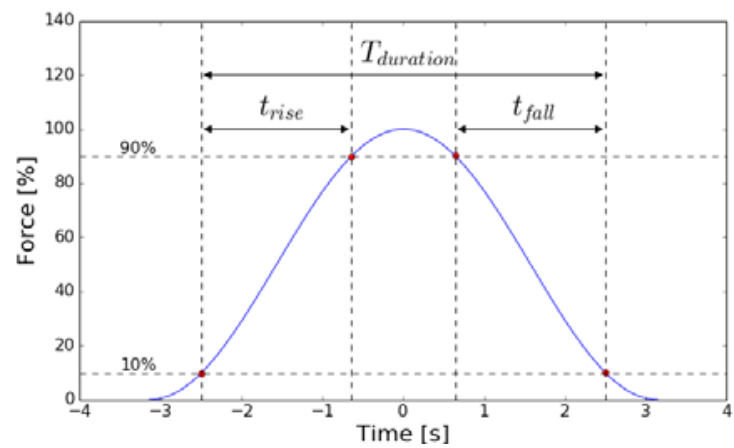

Fig. 7 Example of one repetition
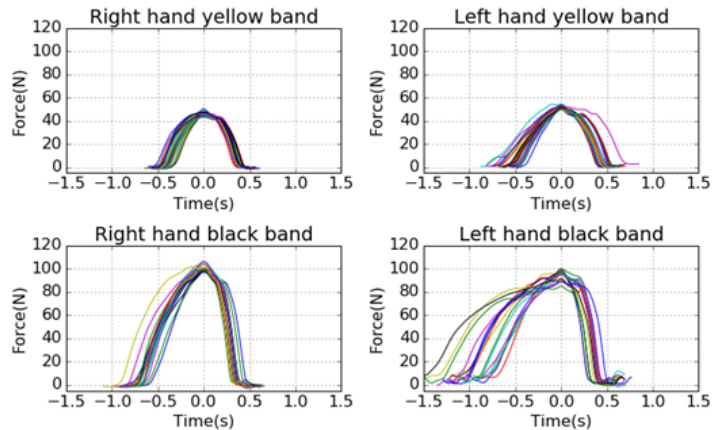

Fig. 8 Segments of each repetition 
Each repetition of 4 sets of test are plotted in Fig. 8. It is clear that in both of the resistance bands, right hand is more stable than left hand in repetition. Especially in the test using the black band, the left hand has significant differences during 30 s of repetitions in the curve. The right hand uses less time to complete a repetition when compared to left hand in both type of the resistance bands. This is of course expected as the subject is right handed.

The comparisons of left hand and right hand in pulling time (Fig. 9) shows that the left hand is easier to be fatigued and the pulling time is increasing more quickly compared to right hand in yellow band. For the black band, the left hand takes more time to lift the band than the right hand, and it has more dispersion than right hand and less number of repetition during the 30 s of the exercise.

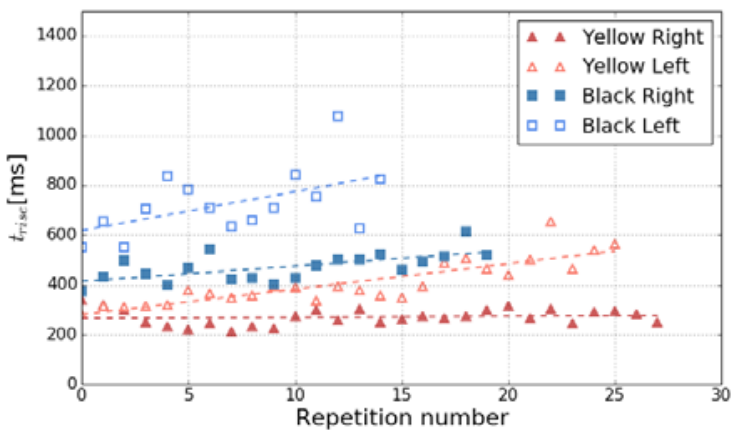

Fig. 9 Pulling up from 10\% to $90 \%$ of peak force

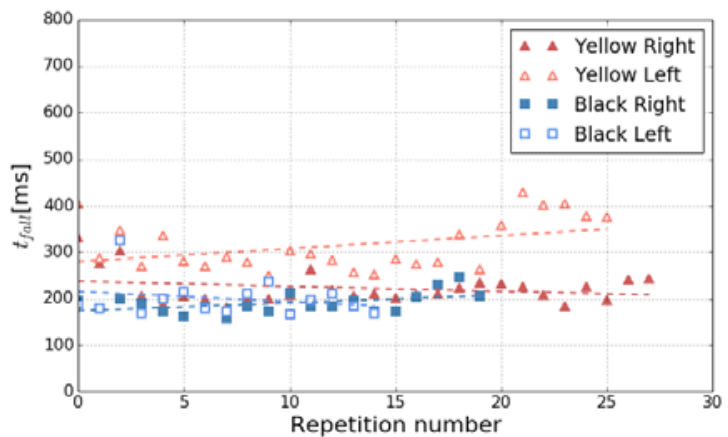

Fig. 10 Falling down from $90 \%$ to $10 \%$ of peak force
In the Fig. 10, the falling time of all the data is almost in the same level and does not change much during the whole exercise. The trendline of the right hand with the yellow band seems to show that the fall time decreases during the exercise. This is however due to a couple of "slow" drops at the beginning of the exercise.

The duration of each repetition (Fig. 11) confirms what already shown by the other graphs.

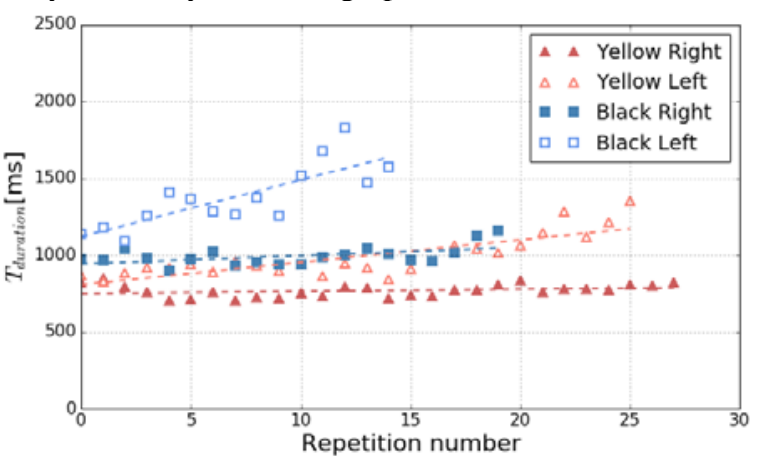

Fig. 11 Duration of each repetition

\section{CONCLUSION}

In this paper, we presented a simple used, compact and portable WBR-SH1 which capable for both experiments sensing and daily exercises monitoring. The resistance band exercises could be assessed objectively by the WBR-SH1 . The same size as the traditional handle makes it more acceptable than other wearable sensing system. The low power design and charging ability allow less maintenance of it.

As the preliminary test, the WBR-SH1 is used as a replacement equipment of the traditional resistance band handle for data collections. The similar size design allows the subject doing exercise with it as the traditional handle. Due to the limitation of time, only force data is analyzed. However, the difference between each test is already shown very clearly. In the future, more experiment and analysis would be performed in both lab-based and off-lab environments with dementia patients.

\section{ACKNOWLEDGMENT}

The authors are grateful to Prof. Eef Hogervorst, Dr. Vassilios Chouliaras, Dr. Bouazza-Marouf, Dr. Stephan Bandelow, and Ms. Jordan Elliott-King for the useful comments. The authors would like to express their gratitude to STMicroelectronics and Life Performance Research for their advice.

\section{REFERENCES}

[1] 'World Alzheimer Report 2015, The Global Impact of Dementia: An analysis of prevalence, incidence, cost and trends - WorldAlzheimerReport2015.pdf'. .

[2] Alzheimer's Society, 'What is dementia?', Alzheimer's Society. [Online]. Available: https://www.alzheimers.org.uk/site/scripts/documents_i nfo.php?documentID=106. [Accessed: 01-Jul-2016].

[3] Alzheimer's Society, 'Factsheet: Assessment and diagnosis', Alzheimer's Society. [Online]. Available: 
https://www.alzheimers.org.uk/site/scripts/download_in fo.php?downloadID=1154. [Accessed: 27-Jun-2016].

[4] E. Hogervorst, 'Exercise to Prevent Cognitive Decline and Alzheimer's disease: For Whom, When, What, and (most importantly) How Much?', J. Alzheimers Dis. Park., vol. 02, no. 03, 2012.

[5] E. H. Angela Clifford, 'What is the Relationship between Higher Levels of Education Delaying Age at Onset of Dementia?', J. Alzheimer's Dis. Park., vol. 03, no. 01, 2013.

[6] D. R. Bassett, 'Device-based monitoring in physical activity and public health research', Physiol. Meas., vol. 33, no. 11, pp. 1769-1783, Nov. 2012.

[7] 'Research - Xsens 3D motion tracking'. [Online]. Available: https://www.xsens.com/research/. [Accessed: 30-Jan-2017].

[8] Z. Lin et al., 'Development of the miniaturized wireless Inertial Measurement Unit WB-4: Pilot test for mastication analysis', in 2010 IEEE/SICE International Symposium on System Integration (SII), 2010, pp. 420425.

[9] 'rtthread_manual.zh.pdf'. [Online]. Available: http://www.rt-thread.org/download/manual/rtthread_ma nual.zh.pdf. [Accessed: 11-Jul-2016].

[10] W. Kong et al., 'Development of a real-time IMU-based motion capture system for gait rehabilitation', in 2013 IEEE International Conference on Robotics and Biomimetics (ROBIO), 2013, pp. 2100-2105. 